\title{
Single photon emission computed tomography: An alternative imaging modality in left ventricular evaluation
}

\author{
Hulya Yalçin ${ }^{1,3}$ \\ Sofiane Maza' \\ Fatih Yalçin ${ }^{2,4}$ \\ 'Department of Nuclear Medicine; \\ ${ }^{2}$ Center for Cardiovascular Research, \\ Charite University, Berlin, Germany; \\ ${ }^{3}$ Department of Nuclear Medicine; \\ ${ }^{4}$ Department of Cardiology, Mustafa \\ Kemal University, Antioch, Turkey
}

\begin{abstract}
Various diagnostic imaging modalities have been used for quantitative left ventricular (LV) parameters. Because of the suboptimal value of the most widely used technology, twodimensional (2D) echocardiography, 3D ultrasonographic imaging has improved accuracy for LV volume and function. Single photon emission computed tomography (SPECT) is another diagnostic method where LV volumetric and functional parameters can be accurately provided by gated myocardial perfusion tomographic slices. First pass radionuclide venticulography is another imaging modality which has some practical limitations. Despite lower ejection fraction (EF) values compared with invasive approach, noninvasive techniques are accurate in determination of normal and depressed EF. Noninvasive techniques with 3D approach including gated SPECT are beneficial for not only global but also regional LV evaluation. It has been mentioned that the slight difference between echocardiography and SPECT could be caused by the diverse population studied. The results of diagnostic stress tests support that SPECT is feasible to use in evaluation of LV volume and functional analysis. Magnetic resonance imaging is an expensive modality to use routinely, but it preserves its importance in selected patients for providing precise LV geometric data.
\end{abstract}

Keywords: geometry, left ventricle, single photon emission computed tomography, volume

\section{Introduction}

Various diagnostic imaging modalities have been used for calculation of left ventricular (LV) volume. Two-dimensional (2D) echocardiography is most widely used technology, but it is suboptimal for detection of LV volume due to the complex LV cavity geometry. In ultrasonographic assessment of LV volumes, current 2D echocardiographic methods to quantitate ventricular performance require image acquisition from standardized scanning planes. LV volume is calculated from assumptions that the ventricular cavity can be represented by individual geometric figures or their combination. Both limitations of acoustic access and distortion of ventricular shape may invalidate these assumptions of standardized imaging planes and geometry, and lead to errors in calculating ventricular volume (Teichholz et al 1976).

Regional diversity of LV may be a difficulty of echocardiographic volume assessment in clinical practice. It has been shown that simplified echocardiographic reconstruction used in conjunction with tissue harmonic imaging and automated endocardial border extraction accurately calculates LV volume and function in patients with regional wall motion abnormalities or distorted LVs caused by ischemic heart disease (Mele et al 2004). 3D ultrasonographic imaging provides improved accuracy for LV volume and function compared with current 2D algorithms (Siu et al 1995; Nosir et al 1996). In $3 \mathrm{D}$ reconstruction, the technique required many cardiac cycles to reconstruct the heart, with considerable artifact due to respiratory and patient movement (Salustri et al 1996). 
An integrated system may provide better agreement between LV volumes calculated by $3 \mathrm{D}$ reconstruction and actual volumes (Handschumacher et al 1993).

Increased LV volumes are associated with high mortality, adverse cardiovascular events, and worse prognosis (Siu et al 1995). Although LV volumes have important prognostic implications, its evaluation is commonly underestimated in practice. If it is evaluated accurately, it provides additional incremental value to risk assessment and guides further therapy. Real-time 3D echocardiography has been compared with TI-201 single photon emission computed tomography (SPECT), cardiac magnetic resonance imaging (MRI) and found to be accurate and reproducible for the measurement of LV volumes for risk assessment in ischemic heart disease and dilated cardiomyopathy (Chan et al 2006).

Determination of regional $\mathrm{LV}$ diversity is the main difficulty not only for ischemic heart disease and volume overload, but for hypertrophied hearts. The novel modality by application of real-time 3D imaging technology can improve understanding of the spatial relations of various cardiac structures and offers the potential for more accurate volumetric quantization (Gopal et al 1992). We previously have used real-time $3 \mathrm{D}$ imaging to evaluate total and segmental $\mathrm{LV}$ volume in patients with LV hypertrophy and have described the details in regional geometry and global LV morphology (Yalçin et al 2000). Using 3D volumetric data, it has become possible to evaluate regional and global LV geometries especially in patients with LV geometric distortion or complex LV morphology. However, 3D approach is not a widely used echocardiographic method in clinical practice.

SPECT is one of the diagnostic modalities to evaluate LV volumetric and functional parameters accurately provided by gated myocardial perfusion tomographic slices. Gated perfusion tomographic slices have been used for volume calculations which includes 2D geometric assumptions and 3D approaches. Geometric modeling has been used for manually 2D calculation of ejection fraction (EF) using gated myocardial perfusion SPECT which has provided acceptable correlation with equilibrium multigated blood-pool and with contrast ventriculography (DePuey et al 1993; Mochizuki et al 1997). In healthy young subjects, post-exercise cardiac changes affect systolic functions detected on gated thallium myocardial perfusion scintigraphy, resulting in a smaller heart size during stress. However, the absence of these findings in the elderly suggests a decrease in contractility with age (Aktaş et al 2005). In addition to aging, decreased contractility may be detected by gated myocardial perfusion SPECT in postischemic stunning due to stress-induced ischemic episode (Johnson et al 1997). Therefore, Johnson and colleagues (1997) suggested that the presence of postischemic reduction in the post-stress EF may be another marker of disease severity and reduced event-free survival.

It was also reported that gated myocardial perfusion SPECT with Tc-99m labeled radiopharmaceutical may provide more objective imaging data than T1-201 because gated SPECT performed with Tc-99m labeled radiopharmaceutical gives better image quality than T1-201 due to higher myocardial count rate. So when objective myocardial function monitoring is needed Tc-99m labeled radiopharmaceutical could be a reasonable method (Hyun et al 2001).

The number of gated frames per cardiac cycle in myocardial gated SPECT may influence the accuracy of both EF and wall thickening. It has been shown that an 8-frame study results in a mismall constant and predictable decrease of 0.04 in EF compared with a 16-frame study (Germano et al 1995). First pass radionuclide venticulography is another imaging modality for EF calculations in which 32 frames per cardiac cycle are used. Despite high frame numbers, first pass radionuclide venticulography has some practical limitations like giving functional data only at the time of injection (Yoshioka et al 1999). Gated myocardial perfusion SPECT has been used with automated technology and has given a satisfactory result (Nichols et al 1997). It has been shown that automated edge-detection algorithm is well correlated with contrast left ventriculogram and MRI (Mochizuki et al 1997; Yoshioka et al 1999).

Planar multigated acquisition is one of the most common used technologies for EF and volume calculation. However, it is difficult to calculate EF of the ventricules due to atrial overlap so SPECT studies are widely used (Lu et al 1994). On the other hand, SPECT studies are not successful in right ventricular EF calculation which is feasibly evaluated by first pass radionuclide ventriculography (Massardo et al 2007). Also in multigated SPECT ventriculography, we could not obtain an idea of the perfusion of the myocardium even if we can begin imaging a few hours later, which differs from first pass radionuclide ventriculography in which imaging must began as soon as possible after injection (Borges-Neto 1997). In the literature, it is stated that gated myocardial perfusion SPECT which gives perfusion in addition to EF and wall motion analysis is superior to gated blood pool SPECT (Meine et al 2004).

Especially in coronary artery disease (CAD) patients, gated myocardial perfusion SPECT gives us more information than other technologies used for EF and volume calculations. With the quantitative gated SPECT (QGS) 
method, even in normal perfusion, we can detect wall motion abnormalities which will influence the survival of CAD patients (Stegger et al 2007). It has been documented that despite small systematic differences, agreement between gated myocardial SPECT and MRI is good over a wide range of volumes and EF values using the QGS method (Schaefer et al 2005).

The variables provided by exercise radionuclide angiocardiography predicts cardiac death in patients with CAD (Jones et al 1991). However, noninvasive assessment of EF usually has been found to be lower than that detected by angiography (Nichols et al 1998; Vourvouri et al 2001; Habash-Bseiso et al 2005). Despite lower EF values by noninvasive assessment, noninvasive techniques are accurate in determination of normal and depressed EF (Habash-Bseiso et al 2005). The quantification of influence of filtering and pixel size parameters on measured LV volumes for gated blood pool SPECT may be beneficial in correction of overestimation (Debrun et al 2005). SPECT has shown a slightly lower EF than that measured by echocardiography (Vourvouri et al 2001; Habash-Bseiso et al 2005), this could be also caused by diverse population studied (Albrechtsson et al 1982).

SPECT with boundary detection method also has shown a good correlation with equilibrium multigated blood-pool and MRI volumes (Faber et al 1988, 1991). Detection of boundaries for volume calculations may be possible in patients who have defect extending more than $25 \%$ of the myocardium (Nichols et al 1997). Border detection becomes more complicated in apical defects, it has been forced the wall to be a smooth connection between noninfarcted portions of the wall (Faber et al 1999). Finally, positron emission tomography from nuclear studies has been used accurately for global and regional detection of LV function (Kanayama et al 2007). It has given an acceptable result for LV volumes and EF in comparison with MRI and angiography (Schaefer et al 2003). Among the noninvasive modalities, MRI is considered the gold standard, because of high accuracy and reproducibility for volume measurements (Barkhausen et al 2001). It has been used for assessment of myocardial thickness and regional myocardial function quantitatively (Pflugfelder et al 1988).

In conclusion, gated myocardial perfusion SPECT is feasible for evaluation of LV volume and functional analysis in routine clinical practice. MRI preserves its importance in selected patients to determine more precise LV geometric data in patients with cardiac diseases, but it is unfortunately more expensive to use routinely.

\section{Disclosure}

The authors report no conflicts of interest in this work.

\section{References}

Aktaş A, Yalçin H, Koyuncu A, et al. 2005. The influence of post-exercise cardiac changes on thallium-gated myocardial perfusion scintigraphy findings in normal subjects. Nucl Med Commun, 26:109-14.

Albrechtsson U, Eskilsson J, Lomsky M, et al. 1982. Comparison of left ventricular ejection fraction assessed by radionuclide angiocardiography, echocardiography and contrast angiocardiography. Acta Med Scand, 211:147-52.

Barkhausen J, Ruehm SG, Goyen M, et al. 2001. MR evaluation of ventricular function: true fast imaging with steady-state precession versus fast low-angle shot cine MR imaging: feasibility study. Radiology, 219:264-9.

Borges-Neto S. 1993. Perfusion and function assessment by nuclear cardiology techniques. Curr Opin Cardiol, 12:581-6.

Chan J, Jenkins C, Khafagi F, et al. 2006. What Is the optimal clinical technique for measurement of left ventricular volume after myocardial infarction? A comparative study of 3-dimensional echocardiography, single photon emission computed tomography, and cardiac magnetic resonance imaging. J Am Soc Echocardiogr, 19:192-201.

Debrun D, Therain F, Nguyen LD, et al. 2005. Volume measurements in nuclear medicine gated SPECT and 4D echocardiography: validation using a dynamic cardiac phantom. Int J Cardiovasc Imaging, 21:239-47.

DePuey EG, Nichols K, Dobrinsky C. 1993. Left ventricular ejection fraction assessed from gated technetium-99m-sestamibi SPECT. J Nucl Med, 34:1871-6.

Faber TL, Stokely EM, Corbett JR. 1988. Surface detection in dynamic tomographic myocardial perfusion images by relaxation labeling. SPLE 1001. Visual Communications and Image Processing, 297-301.

Faber TL, Stokely EM, PeshockRM, et al. 1991. A model-based fourdimensional left ventricular surface detector. IEEE Trans Med Imaging, 10:321-9.

Faber TL, Cooke DR, Folks RD, et al. 1999. Left ventricular function and perfusion from gated SPECT perfusion images: An integrated method. J Nucl Med, 40:650-9.

Germano G, Kiat H, Kavanagh PB, et al. 1995. Automatic quantification of ejection fraction from gated myocardial perfusion SPECT. $J$ Nucl Med, 36:2138-47.

Gopal AS, King DL, Katz J, et al. 1992. Three dimensional echocardiographic volume computation by polyhedral surface reconstruction: in vitro validation and comparison to magnetic resonance imaging. $J \mathrm{Am} S o c$ Echocardiogr, 5:115-24.

Habash-Bseiso DE, Rokey R, Berger CJ, et al. 2005. Accuracy of noninvasive ejection fraction measurement in a large community-based clinic. Clin Med Res, 3:75-82.

Handschumacher MD, Lethor JP, Siu SC, et al. 1993. A new fully integrated system for three-dimensional echocardiographic reconstruction: validation for ventricular volume with application in human subjects. $J \mathrm{Am}$ Coll Cardiol, 21:743-53.

Hyun IY, Kwan J, Park KS, et al. 2001. Reproducibility of Tl-201 and Tc-99m sestamibi gated myocardial perfusion SPECT measurement of myocardial function. J Nucl Cardiol, 8:182-7.

Johnson LL, Verdesca SA, Aude WY, et al. 1997. Postischemic stunning can affect left ventricular ejection fraction and regional wall motion on poststress gated sestamibi tomograms. J Am Coll Cardiol, 30:1641-8.

Jones RH, Johnson SH, Bigelow C, et al. 1991. Exercise radionuclide angiocardiography predicts cardiac death in patients with coronary artery disease. Circulation, 84:152-8.

Kanayama S, Matsunari I, Kajinami K. 2007. Comparison of gated N-13 ammonia PET and gated Tc-99m sestamibi SPECT for quantitative analysis of global and regional left ventricular function. J Nucl Cardiol, 14:680-7. 
Lu P, Liu X, Shi R, et al. 1994.Comparison of tomographic and planar radionuclide ventriculography in the assessment of regional left ventricular function in patients with left ventricular aneurysm before and after surgery. J Nucl Cardiol, 1:537-45.

Massardo T, Jaimovich R, Lavados H, et al. 2007. Comparison of radionuclide ventriculography using SPECT and planar techniques in different cardiac conditions. Eur J Nucl Med Mol Imaging, 34:1735-46.

Mele D, Teoli R, Cittanti C, et al. 2004. Assessment of left ventricular volume and function by integration of simplified 3D echocardiography, tissue harmonic imaging and automated extraction of endocardial borders. Int J Cardiovasc Imaging, 20:191-202.

Meine TJ, Hanson MW, Borges-Neto. 2004. The additive value of combined assessment of myocardial perfusion and ventricular function studies. J Nucl Med, 45:1721-4.

Mochizuki T, Murase K, Tanaka H, et al. 1997. Assessment of left ventricular volume using ECG-gated SPECT with technetium-99m-MIBI and technetium-99m tetrofosmin. J Nucl Med, 38:53-7.

Nichols K, DePuey EG, Rozanski A, et al. 1997. Image enhancement of severely hypoperfused myocardia for computation of tomographic ejection fraction. J Nucl Med, 38:1411-17.

Nichols K, Tamis J, DePuey EG, et al. 1998. Relationship of gated SPECT ventricular function parameters to angiographic measurements. $J \mathrm{Nucl}$ Cardiol, 5:295-303.

Nosir YF, Fioretti PM, Vletter WB, et al. 1996. Accurate measurement of left ventricular ejection fraction by three-dimensional echocardiography. A comparison with radionuclide angiography. Circulation, 94:460-66.

Pflugfelder PW, Sechtem UP, White RD, et al. 1988. Quantification of regional myocardial function by rapid cine MR imaging. AJR Am J Roentgenol, 150:523-9.

Salustri A, Kofflard MJ, Roelandt JR, et al. 1996. Assessment of left ventricular outflow in hypertrophic cardiomyopathy using anyplane and paraplane analysis of three-dimensional echocardiography. Am J Cardiol, 78:462-8.
Schaefer WM, Lipke CSA, Nowark B, et al. 2003. Validation of an evaluation routine for left ventricular volumes, ejection fraction and wall motion from gated cardiac FDG PET: a comparison with cardiac magnetic resonance imaging. Eur J Nucl Med Mol Imaging, 30:545-53.

Schaefer WM, Lipke CS, Standke D, et al. 2005. Quantification of left ventricular volumes and ejection fraction from gated $99 \mathrm{mTc}-\mathrm{MIBI}$ SPECT: MRI validation and comparison of the Emory Cardiac Tool Box with QGS and 4D-MSPECT. J Nucl Med, 46:1256-63.

Siu SC, Levine RA, Rivera JM, et al. 1995. Three-dimensional echocardiography improves noninvasive assessment of left ventricular volume and performance. Am Heart J, 130:812-22.

Stegger L, Lipke CS, Kies P, et al. 2007. Quantification of left ventricular volumes and ejection fraction from gated 99mTc-MIBI SPECT: validation of an elastic surface model approach in comparison to cardiac magnetic resonance imaging, 4D-MSPECT and QGS. Eur J Nucl Med Mol Imaging, 34:900-9.

Teichholz LE, Kreulen T, Herman MV, et al. 1976. Problems in echocardiographic volume determinations: echocardiographic-angiographic correlations in the presence or absence of asynergy. Am J Cardiol, 37:7-11.

Vourvouri EC, Poldermans D, Bax JJ, et al. 2001. Evaluation of left ventricular function and volumes in patients with ischaemic cardiomyopathy: gated single-photon emission computed tomography versus two dimensional echocardiography. Eur J Nucl Med, 28:1610-15.

Yalçin F, Shiota T, Odabashian J, et al. 2000. Comparison by real-time three-dimensional echocardiography of left ventricular geometry in hypertrophic cardiomyopathy versus secondary left ventricular hypertrophy. Am J Cardiol, 85:1035-8.

Yoshioka J, Hasegawa S, Yamaguchi H, et al. 1999. Left ventricular volumes and ejection fraction calculated from quantitative electrocardiographic- gated $99 \mathrm{mTc}-$ Tetrofosmin myocardial SPECT. J Nucl Med, 40:1693-8. 